\title{
Pentaquarks in the medium in the quark-meson coupling model
}

\author{
P.K. Panda and C. Providência \\ Centro de Física Teórica - Dep. de Física - Universidade de Coimbra - P-3004 - 516 Coimbra - Portugal \\ D.P. Menezes \\ Depto de Fúsica - CFM - Universidade Federal de Santa Catarina \\ Florianópolis - SC - CP. 476 - CEP 88.040 - 900 - Brazil and \\ School of Physics - University of Sydney - NSW - 2006 - Australia
}

(Dated: November 6, 2018)

\begin{abstract}
We calculate the properties of the pentaquarks $\Theta^{+}$and $\Xi^{--, 0}$ in symmetric nuclear matter using the quark meson coupling model (QMC). The stability of the $\Theta^{+}$in the medium with respect to the channel $\Theta^{+} \rightarrow N K^{+}$is discussed.
\end{abstract}

PACS number(s): 36.10.-k,24.10.Jv, 21.65.+f

\section{INTRODUCTION}

The possible existence of pentaquarks was first proposed a long time ago [1] but the subject regained theoretical interest after such exotic states were indeed measured [2, 3]. In the recent months many works have been dedicated to the calculation of pentaquark properties [6, 7, 8, 9, 10], a theoretical pioneer work being the one that classified a narrow strange pentaquark with positive parity as the lowest state of an $S U(3)$ decuplet within a chiral soliton model 11.

Three of the possible pentaquarks are the $\Theta^{+}$[2, 3] containing the quarks uudd $\bar{s}$ with mass 1540 $\mathrm{MeV}, S=+1$, zero isospin $(I=0)$ and positive charge, the $\Xi^{--}$, formed by $d s s d \bar{u}, S=-2, I=3 / 2$ and the $\Xi^{0}$ constituted of quarks $d s s u \bar{d}, S=-2, I=3 / 2$ and neutral, the last two with masses equal to $1862 \mathrm{MeV}$, detected in [4] but not confirmed in other experiment [5].

Another topic of great interest in the recent literature is the in-medium modification of baryon properties, mainly their masses and decay widths 12, 13, 14, 15.

In the present work we use the quark-meson coupling model (QMC) [16, 17] in order to obtain the effective masses of the pentaquarks $\Theta^{+}$and $\Xi^{--, 0}$ in a self-consistent way with the nucleon mass. We fix the nucleon radius and determine the pentaquark radius considering the same bag constant of the nucleon. We compare the chemical potential of the pentaquarks determined in this way with the chemical potential of the nucleon plus kaon system as a function of the density. A comparison with the results obtained within the non-linear Walecka model (NLWM) [19] is drawn.

\section{THE QUARK-MESON COUPLING MODEL FOR PENTAQUARKS}

In the QMC model, the baryons are assumed to be static spherical MIT bags in which quarks interact with the scalar and vector fields, $\sigma, \omega$ and $\rho$ and these fields are treated as classical fields in the mean field approximation. In the present work we treat nucleons and pentaquarks within the same formalism, the differences arising from the number and flavor of the constituent quarks. We consider both the $\Theta^{+}$and the $\Xi^{--, 0}$ in nuclear matter and assume that non-strange mesons do not couple with the $s, \bar{s}$ quarks.

The quark field, $\psi_{q}(x)$, inside the bag satisfies the equation of motion:

$$
\left[i \not \partial-\left(m_{q}^{0}-g_{\sigma}^{q} \sigma_{0}\right)-\gamma^{0}\left(g_{\omega}^{q} \omega_{0}+\frac{1}{2} g_{\rho}^{q} \tau_{3 q} b_{03}\right)\right] \psi_{q}(x)=0, \quad q=u, d, s,
$$

where $\sigma_{0}, \omega_{0}$ and $b_{03}$ are the classical meson fields for $\sigma, \omega$ and $\rho$ mesons. $m_{q}^{0}$ is the current quark mass, $\tau_{3 q}$ is the third component of the Pauli matrices and $g_{\sigma}^{q}, g_{\omega}^{q}$ and $g_{\rho}^{q}$ are the quark couplings with $\sigma, \omega$ and $\rho$ mesons.

The quark energy is

$$
\epsilon_{q}=\Omega_{q}+R_{B}\left(g_{\omega}^{q} \omega+\frac{1}{2} g_{\rho}^{q} \tau_{3 q} \rho_{03}\right)
$$


with $\Omega_{q} \equiv \sqrt{x_{q}^{2}+\left(R_{B} m_{q}^{*}\right)^{2}}, m_{q}^{*}=m_{q}^{0}-g_{\sigma}^{q} \sigma, R_{B}$ is the bag radius of the baryon. The energy of a static bag describing the baryon $B$, can be expressed as

$$
E_{B}^{\mathrm{bag}}=\sum_{q} n_{q} \frac{\Omega_{q}}{R_{B}}-\frac{Z_{B}}{R_{B}}+\frac{4}{3} \pi R_{B}^{3} B_{B}
$$

where $Z_{B}$ is a parameter which accounts for zero-point motion and $B_{B}$ is the bag constant. The set of parameters used in the present work is given in [20] for the bag value $B_{B}^{1 / 4}=210.854 \mathrm{MeV}$, $m_{u}^{0}=m_{d}^{0}=5.5 \mathrm{MeV}$ and $m_{s}^{0}=150 \mathrm{MeV}$. The effective mass of the nucleon and the pentaquark at rest is taken to be

$$
M_{B}^{*}=E_{B}^{\mathrm{bag}} .
$$

The equilibrium condition for the bag is obtained by minimizing the effective masses $M_{B}^{*}$ with respect to the bag radius

$$
\frac{\partial M_{B}^{*}}{\partial R_{B}^{*}}=0
$$

All the above expressions are valid for the nucleons and the pentaquarks. We have considered the same bag constant $B_{B}$ for both. The radius and the $Z_{B}$ parameter for the pentaquark are then obtained from equations (2.4) (for the vacuum) and (2.5).

It is not clear if the spin parity of the pentaquark $\Theta^{+}$is $J^{P}=1 / 2^{+}$or $J^{P}=1 / 2^{-}$. Constituent quark models predict the first value [6] and chiral symmetric models the second [1]]. Within the QMC model, we may have a positive parity state if one of the quarks occupies a $p$ state. However, this is not the lowest energy state. In the following we also consider this possibility.

For simplicity, we assume that the bag describing the nucleon remains in a spherical shape with radius $R_{B}$. The single-particle energies in units of $R^{-1}$ are given as [21]

$$
\epsilon_{q}=\Omega_{q}^{n \kappa} \pm R_{B}\left(g_{\omega}^{q} \omega+\frac{1}{2} g_{\rho}^{q} \tau_{3 q} \rho_{03}\right)
$$

for quarks and antiquarks, with $\Omega_{q}^{n \kappa}=\sqrt{x_{n \kappa}^{2}+R_{B}^{2} m_{q}^{* 2}}$. The eigenvalues $x_{n \kappa}$ for the state characterized by $n$ and $\kappa$ are determined by the boundary condition at the bag surface, $i \gamma \cdot n \psi_{q}^{n \kappa}=\psi_{q}^{n \kappa}$. We get for the total energy

$$
E_{B}^{\mathrm{bag}}=\sum_{q} \frac{\Omega_{q}^{n \kappa}}{R_{B}}-\frac{Z_{B}}{R_{B}}+\frac{4}{3} \pi R_{B}^{3} B_{B}
$$

For the QMC model the equations of motion for the meson fields in uniform static matter are the ones usually given in the literature [16, 17, 20]. For the necessary self-consistent treatment, one has to determine

$$
\frac{\partial M_{B}^{*}(\sigma)}{\partial \sigma}=\frac{\partial E_{B}^{\mathrm{bag}}}{\partial \sigma}
$$

Notice that, although we have included the $\rho$ meson in the equations above, we shall restrict ourselves to the calculation of symmetric nuclear matter. Moreover, as we have opted to use identical masses for the $u$ and $d$ quarks, the pentaquarks $\Xi^{0}$ and $\Xi^{--}$are indistinguishable.

Boosting the bags we get the dispersion relation for the pentaquarks

$$
\mu_{\Theta}=\sqrt{M_{B}^{* 2}+\mathbf{k}^{2}}+g_{\omega} \omega_{0} .
$$

We also calculate the optical potential of a pentaquark in symmetric nuclear matter, which we define as

$$
V_{B}=M_{B}^{*}-M_{B}+g_{\omega B} \omega_{0} .
$$

In the numerical calculations we have used [20] $g_{\sigma}^{q}=5.957, g_{\sigma N}=3 g_{\sigma}^{q} S_{N}(0)=8.58, g_{\omega N}=8.981$, $g_{\rho N}=8.651$ with $g_{\omega N}=3 g_{\omega}^{q}$ and $g_{\rho N}=g_{\rho}^{q}$, with the standard values for the meson masses, $m_{\sigma}=550$ $\mathrm{MeV}, m_{\omega}=783 \mathrm{MeV}$ and $m_{\rho}=770 \mathrm{MeV}$. Note that in our approach the $s$-quark is unaffected by the $\sigma, \omega$ and $\rho$ mesons i.e. $g_{\sigma}^{s}=g_{\omega}^{s}=g_{\rho}^{s}=0$. The pentaquark couplings are given by $g_{\omega \Theta}=\frac{4}{3} g_{\omega N}$, 
$g_{\rho \Theta}=g_{\rho N}$. In Table $\amalg$ we give the values of $Z$ for both $\Theta^{+}$and $\Xi^{--, 0}$ considering the radius of the nucleon 0.6 and $1.0 \mathrm{fm}$ and the three different cases: a) all quarks in a state; b) quarks $u$ or $d$ in a $p$ state; c) quarks $\bar{s}$ or $s$ in a $p$ state.

In the next section we compare the QMC results obtained in the present work with results obtained from the NLWM model [19] with the NL3 parametrization [22]. In this model the effective mass of a baryon is $M_{B}^{*}=M_{B}-g_{\sigma}^{B} \sigma$ [24]. Using quark counting arguments and considering that the $\sigma, \omega$ mesons do not couple to the $s$-quark we take

$$
g_{i \Theta}=\frac{4}{3} g_{i N}, \quad g_{i \Xi}=g_{i N}, \quad i=\sigma, \omega
$$

\section{RESULTS}

The in-medium results obtained with the QMC model for the pentaquarks are next displayed and compared with the ones obtained with the NLWM.

In figure 1 we show the effective masses for the $\Theta^{+}$and the $\Xi^{--, 0}$ for two different values of the bag radius. For the larger value of the bag radius, the decrease of the effective masses is less pronounced for both pentaquarks. Our results do not agree with the ones calculated in [9], where, however, the nucleon saturation data of [17] are also not reproduced.

The $\Theta^{+}$effective masses always decrease more with density than $\Xi^{--, 0}$, either within the QMC or the NLWM framework. This is due to the fact that the $s$-quark does not couple to the $\sigma$-meson and $\Xi^{--, 0}$ have two $s$-quarks. For each pentaquark, one can see that the effective masses always decrease more within the NLWM than within the QMC model. This effect was already known for the effective mass of the nucleon: for the the saturation density, QMC gives an effective mass $\sim 0.8 M_{N}$ while the NLWM predicts $\sim 0.7 M_{N}$.

In Fig. 2] we replot the $\Theta^{+}$and $\Xi^{--, 0}$ effective masses in separate graphs. We include the results obtained considering a quark in a $p_{3 / 2}$ state. A smaller decrease of the effective mass with density is observed, but the mass difference with respect to the solution with all quarks in a $s$ state is small.

We have also verified that isospin asymmetry of nuclear matter has little effect on the effective mass of the pentaquarks: for a proton fraction $y_{p}=0.1$ there is a small increase of the effective mass with density with respect to the symmetric matter value. However, at $3 \rho_{0}$ the difference is only $\sim 1 \%$ the symmetric matter value.

We have calculated the radii for three different particles, namely, the nucleon, the kaon and the pentaquark $\Theta^{+}$fixing the nucleon radius as $0.6 \mathrm{fm}$ and choosing for the other particles the same bag constant. It is known that QMC predicts a small reduction of the baryon radius with density [18]. In Fig. 3 we plot, in units of the vacuum nucleon radius, the radius of these three particles. One can see that the $\Theta^{+}$radius is only $16 \%$ larger than the nucleon radius and presents the same behavior with density.

In figure 4 we have shown the optical potentials obtained within both models discussed in the present work. Within the QMC model we get an optical potential of $\sim-75 \mathrm{MeV}$ for the $\Theta^{+}$pentaquark and $\sim-50 \mathrm{MeV}$ for the $\Xi$ pentaquarks. These values are just a bit smaller than the ones predicted with the NLWM and are compatible with values obtained in [23], -70 to $-120 \mathrm{MeV}$ obtained from the calculus of the $\Theta^{+}$self-energy tied to a two-meson cloud. In 10] a value $-90 \mathrm{MeV} \leq U \leq-40$ $\mathrm{MeV}$ was obtained within a QCD sum rule calculation and similar numbers were obtained within a relativistic mean field calculation in [8]. Taking different values for the bag radius has a negligible effect on the potential.

In order to understand under which conditions the $\Theta^{+}$is stable in the medium with respect to the channel $N K$ we have calculated within the QMC model the chemical potential of the pentaquark given by Eq. (2.9), with the chemical potential of the system nucleon plus kaon. For the kaon we use the results of [26]. We consider a pentaquark in its rest frame and suppose it decays, $\Theta^{+} \rightarrow N^{+} K^{+}$. In the matter, due to the Pauli principle the nucleon must have a momentum equal to the Fermi momentum. The kaon will have the same momentum if we don't take into account the effect of the medium. Taking into account the medium, momentum may be shared by any other nucleons and the kaon will come out with any momentum. In Fig. 5 we plot the chemical potential of the $\Theta^{+}$at rest, and the chemical potential of the system $N K^{+}$in two different cases: a) both particles with $p=p_{F}$; b) the nucleon with $p=p_{F}$ and the kaon with a zero momentum. Considering the second possibility the pentaquark $\Theta^{+}$will not be stable in matter. If we consider the first possibility the pentaquark $\Theta^{+}$is stable for densities $>1.5 \rho_{0}$.

However, according to [25], the $K^{+}$feels a weak repulsive potential and the $K^{-}$a strong attractive one. In order to obtain a value approximately equal to $10 \mathrm{MeV}$ for the $K^{+}$potential in the present formalism, we must rescaled the coupling $g_{\omega K}$ as $1.625 g_{\omega K}$ for the nucleon bag radius $R_{N}=0.6$ 
$\mathrm{fm}$ and the kaon bag radius $R_{K}=0.457 \mathrm{fm}$ (see [26, 27]. Taking this value we get different results for the chemical potential of the system nucleon plus kaon represented by thin dashed $\left(p_{K}=0\right)$ and dashed-dot $\left(p_{K}=p_{F}\right)$ lines in Fig. 5 In this case we could have stable pentaquarks, respectively, for densities larger than $\rho_{0}\left(2.5 \rho_{0}\right)$ if we consider the process $\Theta^{+} \rightarrow N+K^{+}\left(\Theta^{+} N \rightarrow N+N+K^{+}\right)$.

In this short note we have calculated the behavior of the $\Theta^{+}$and $\Xi^{--, 0}$ pentaquarks in the medium according to the predictions of the QMC model. In particular we have calculated the effective mass and the optical potential of those particles as a function of density. It was shown, by comparing the chemical potentials of the $\Theta^{+}$and the $N K^{+}$system that $\Theta^{+}$is stable against the decay $\Theta^{+} \rightarrow N K^{+}$for $\rho>1.5 \rho_{0}$. However, taking into account the medium, $\Theta^{+}$is not stable even at high densities. However, these limits are sensitive to the kaon optical potential and they could be lowered, respectively, for $\rho_{0}$ and $2.5 \rho_{0}$ if we choose a $g_{K \omega}$ coupling constant which reproduces the $K^{+}$optical potencial accepted value of $10 \mathrm{MeV}$ at saturation density.

There have been recent calculations of the pentaquarks in the medium using the same model $[8,9]$. Notice, however, that in [8] the properties of the $\Theta^{+}$have not been calculated in a self consistent way in the QMC model and in $9 \mid$ the data obtained for the nucleon properties are not in accordance with the data in the literature [16, 17, 18].

\section{ACKNOWLEDGMENTS}

This work was partially supported by Capes (Brazil) under process BEX 1681/04-4, CAPES (Brazil)/GRICES (Portugal) under project 100/03 and FEDER/FCT (Portugal) under projects POCTI/FP/FNU/50326/2003 and POCTI/FIS/451/94. D.P.M. would like to thank the friendly atmosphere at the Reserch Centre for Theoretical Astrophysics, Sydney University, where this work was partially done.

[1] H. Hogaasen, P. Sorba, Nucl. Phys. B 145 (1978) 119; M. de Crombrugghe, H. Hogaasen and P. Sorba, Nucl. Phys. 347 B 156 (1978); D. Strottman, Phys. Rev. D 20 (1979) 748.

[2] T. Nakano et. al., LEPS Collaboration, Phys. Rev. Lett. 91 (2003) 012202.

[3] S. Stepanyan et. al., CLAS Collaboration, Phys. Rev. Lett. 91 (2003) 252001; J. Barth et. al., SAPHIR Collaboration, Phys. Lett. B 572 (2003) 127.

[4] C. Alt et.al., NA49 Collaboration, Phys. Rev. Lett. 92 (2004) 042003.

[5] M. I. Adamovich et al, WA89 Collaboration, hep-ex/0405042

[6] Fl. Stancu and D.O. Riska, Phys. Lett. B 575 (2003) 242; Fl. Stancu, Phys. Lett. B 595 (2004) 269.

[7] H. Shen and H. Toki, nucl-th/0410072

[8] X.H. Zhong, Y.H. Tan, G.X. Peng, L. Li and P.Z. Ning, Phys. Rev. C 71 (2005) 015206; X.H. Zhong and P.Z. Ning, nucl-th/0501064

[9] C.Y. Ryu, C.H. Hyun, J.Y. Lee and S.W. Hong, nucl-th/0506052

[10] F.S. Navarra, M. Nielsen and K. Tsushima, Phys. Lett. B 606 (2005) 335.

[11] D. Diakonov, V. Petrov, M. Polyakov, Z. Phys. A 359 (1997) 305.

[12] T. Hatsuda and T. Kunihiro, Prog. Theor. Phys. 74, (1985) 765; M. C. Ruivo and C. A. de Sousa, Phys. Lett.B 385 (1996) 39; C. A. de Sousa and M. C. Ruivo, Nucl. Phys. A 625 (1997) 713; C. Ruivo, C. Sousa e C. Providência, Nucl. Phys. A 651 (1999) 59.

[13] D. Trnka et al, CBELSA/TAPS Collaboration, nucl-ex/0504010

[14] J. Adams et al., Phys. Rev. Lett. 92 (2004) 092301; K. Suzuki et a., Phys. Rev. Lett. 92 (2004) 072303; M. Naruki et al. nucl-ex/0504016

[15] S.S. Avancini and D.P. Menezes, nucl-th/0507005

[16] P. A. M. Guichon, Phys. Lett. B 200, 235 (1988).

[17] K. Saito and A.W. Thomas, Phys. Lett. B 327 (1994) 9; 335 (1994) 17; Phys. Rev. C 52, 2789 (1995);

[18] P.A.M. Guichon, K. Saito, E. Rodionov, and A.W. Thomas, Nucl. Phys. A601 349 (1996).

[19] J.D. Walecka, Ann. Phys. 83, 491 (1974); B.D. Serot and J.D. Walecka, Adv. Nucl. Phys. 16, 1 (1986).

[20] P.K. Panda, D.P. Menezes and C. Providência, Phys. Rev. C69 025207 (2004).

[21] P.K. Panda, A. Mishra, J.M. Eisenberg and W. Greiner, Phys. Rev. C56 3134 (1997); P.K. Panda, R. Sahu and C. Das, Phys. Rev. C60 038801 (1999); P. K. Panda, G. Krein, D.P. Menezes, C. Providenĉia, Phys. Rev. C68 015201 (2003).

[22] G. A. Lalazissis, J. König and P. Ring, Phys. Rev. C 55, 540 (1997).

[23] D. Cabrera, Q.B. Li, V.K. Magas, E. Oset, M.J. Vicente Vacas, Phys.Lett. B 608, 231 (2005).

[24] N. K. Glendenning, Compact Stars, Springer-Verlag, New-York, (2000).

[25] G.Q.Li, C.-H. Lee and G.E. Brown, Phys. Rev. Lett 79, 5214 (1997).

[26] D.P. Menezes, P.K. Panda, and C. Providência, Phys. Rev. C (2005), in press; astro-ph/0506196

[27] K. Tsushima, K. Saito, A.W. Thomas and S.V. Wright, Phys. Lett. B 429239 (1998). 
TABLE I: Bag constant and $Z_{0}$ in free space are given in the table. The mass of the quark $m_{u}=m_{d}=5.5$ $\mathrm{MeV}$ and $m_{s}=150 \mathrm{MeV}$. The constants are fixed for Nucleon mass $939 \mathrm{MeV}, \Theta^{+}$mass $1540 \mathrm{MeV}$ and $\Xi^{--, 0}=1862 \mathrm{MeV}$. The $\sigma$ and $\omega$ nucleon couplings are fixed from the saturation properties of nuclear matter i.e. $E_{B}=15.7 \mathrm{MeV}$ at $\rho_{0}=0.15 \mathrm{fm}^{-3}$

\begin{tabular}{lcccccc}
\hline \hline$R_{N}(\mathrm{fm})$ & $g_{\sigma N}^{q}$ & $g_{\omega N}$ & $B_{\Theta}^{1 / 4}$ & $Z_{\Theta}$ & $Z_{\Xi}$ & \\
\hline 0.6 & 5.957 & 8.981 & 210.854 & 6.3765 & 5.4869 & all quarks in $s$ state \\
& & & 7.5359 & 6.6445 & $u$ or $d$-quark in $p$ state \\
& & & 7.4991 & 6.6052 & $s$-quark in $p$ state \\
\hline 1.0 & 5.519 & 7.622 & 143.745 & 3.8428 & 2.3919 & all quarks in $s$ state \\
& & & 4.9969 & 3.5479 & $u$ or $d$-quark in $p$ state \\
& & & 4.9298 & 3.4749 & $s$-quark in $p$ state \\
\hline \hline
\end{tabular}

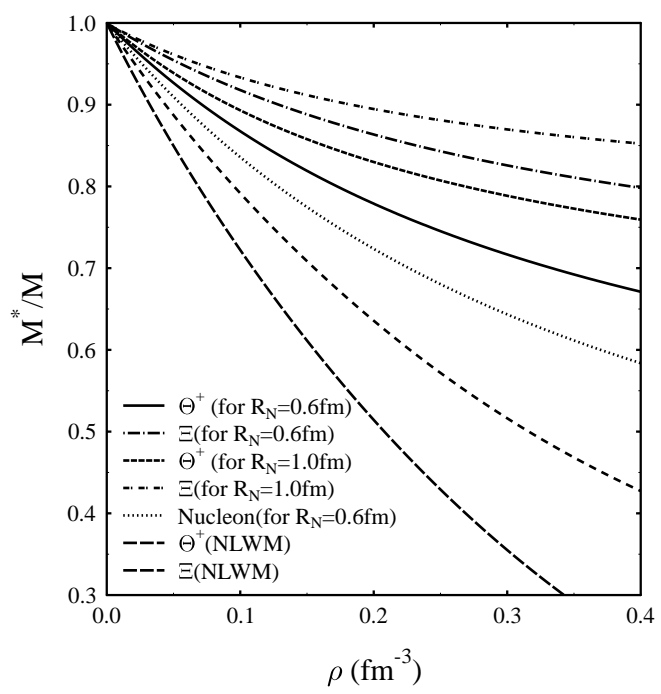

FIG. 1: Effective masses of the pentaquarks $\Theta^{+}$and $\Xi^{--, 0}$ within the QMC and the NLWM models.
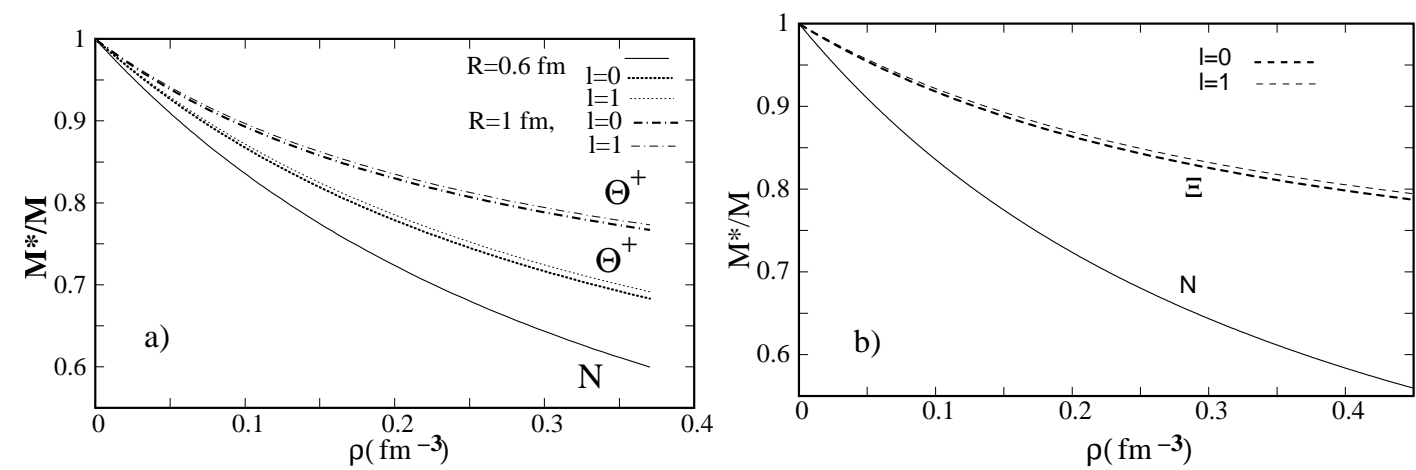

FIG. 2: Effective masses of the pentaquarks $\Theta^{+}$and $\Xi^{--, 0}$ 


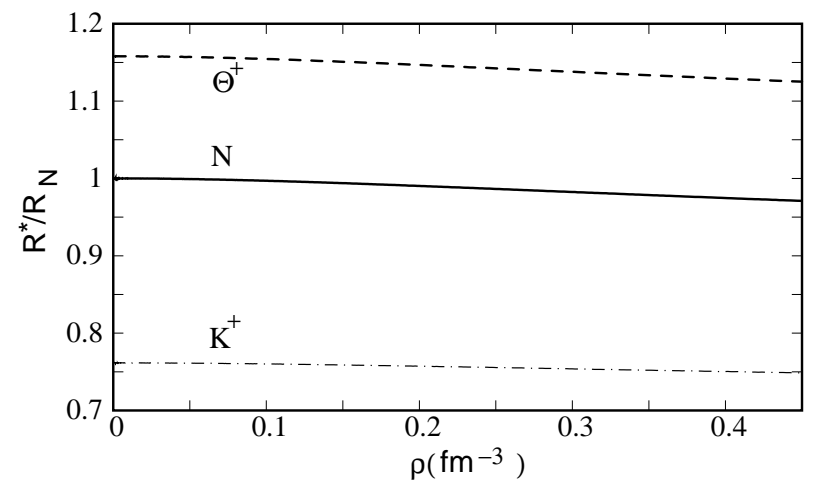

FIG. 3: Radius of the $\Theta^{+}$, nucleon and kaon as a function of density.
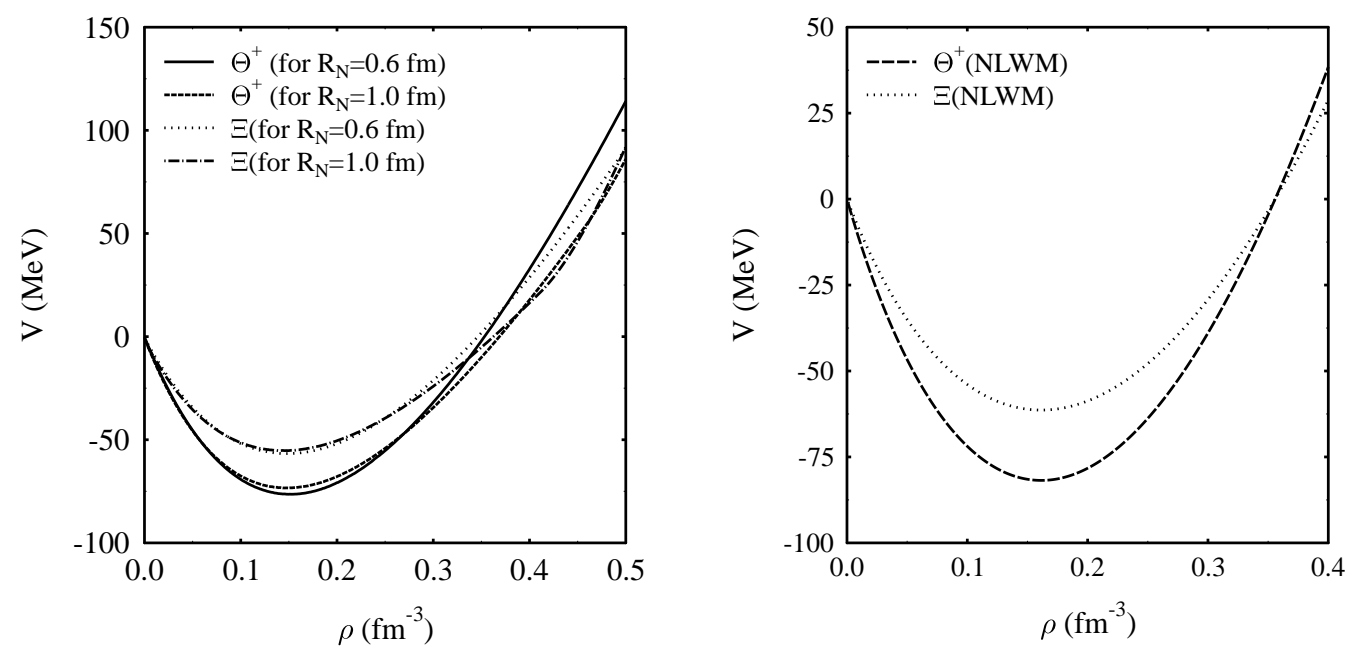

FIG. 4: Optical potentials obtained with the QMC and the NLWM.

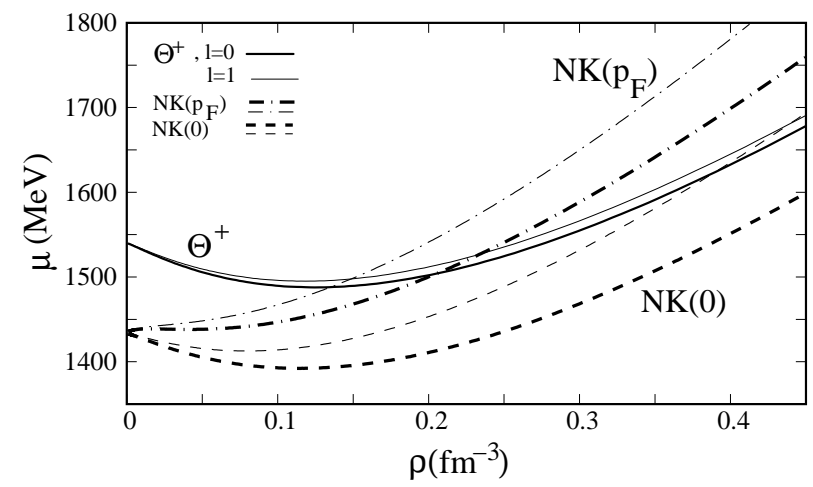

FIG. 5: Chemical potentials of the $\Theta^{+}$, and the $N K^{+}$system. 